\title{
Vulnerabilidad de estructuras en base a los periodos de vibración
}

\author{
Andrea Cevallos ${ }^{\mathrm{a}}$, Angie Burbano ${ }^{\mathrm{a}}$, Ronny Moreano ${ }^{\mathrm{a}}$, Pablo Caiza ${ }^{\mathrm{b}}$ \\ ${ }^{a}$ Estudiante, Carrera de Ingeniería Civil, Universidad de las Fuerzas Armadas ESPE, Sangolquí Ecuador. \\ ${ }^{\mathrm{b}}$ Profesor, Departamento de Ciencias de la Tierra y la Construcción, Universidad de las Fuerzas Armadas ESPE, Sangolquí \\ Ecuador. \\ aecevallos7@espe.edu.ec, aeburbano@espe.edu.ec,rjmoreano@espe.edu.ec, pecaiza1@espe.edu.ec
}

\begin{abstract}
Resumen-Se estudia el cociente, alto de la edificación para periodo de vibración, como un indicador de la vulnerabilidad de edificaciones en el Ecuador. En primer lugar, se lo calcula en modelos analíticos de 2 y 3 pisos. Debido a la dificultad en modelar la mampostería, se sugiere la estimación del periodo de vibración por medios experimentales. Se describe una metodología que usa equipos y software ya disponibles en el Ecuador. Se concluye que este cociente $H / T$ es un parámetro relativamente confiable para determinar la vulnerabilidad estructural. Finalmente, para reducir costos, se propone el uso de acelerómetros tipo Arduino.
\end{abstract}

Palabras Claves-Vulnerabilidad estructural, períodos de vibración, acelerómetros.

Abstract-The quotient, building height to vibration period, is studied as an indicator of the vulnerability of buildings in Ecuador. First, it is calculated in 2- and 3-story analytical models. Due to the difficulty in modeling the masonry, it is suggested to estimate the period of vibration by experimental means. A methodology is described that uses equipment and software already available in Ecuador. It is concluded that this $\mathrm{H}$ / $\mathrm{T}$ ratio is a relatively reliable parameter to determine structural vulnerability. Finally, to reduce costs, the use of Arduino type accelerometers is proposed.

Keywords-Structural vulnerability, vibration periods, accelerometers.

\section{INTRODUCCIÓN}

El sismo de 16 de abril de 2016, en Pedernales, Ecuador, mostró la elevada vulnerabilidad de las edificaciones aporticadas que se usan tradicionalmente en el país. Sobre todo se presentaron fallas en la mampostería, tanto dentro como fuera de su plano, como se muestra en la Figura 1.

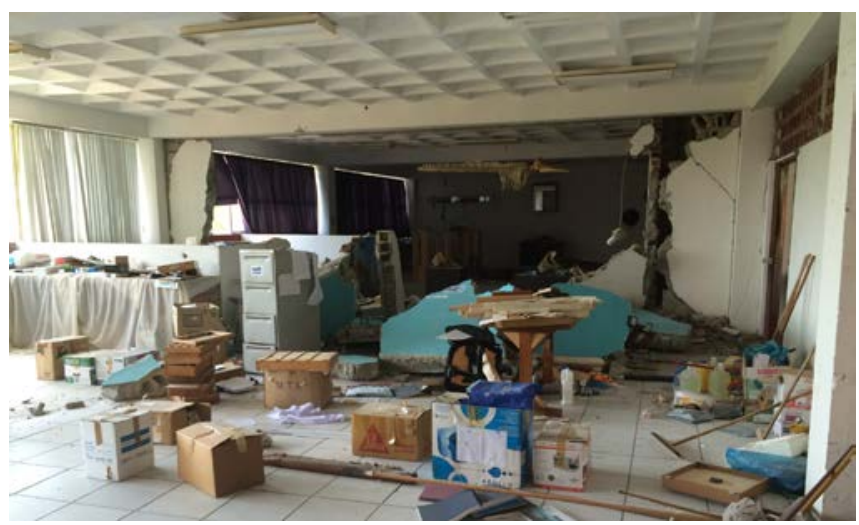

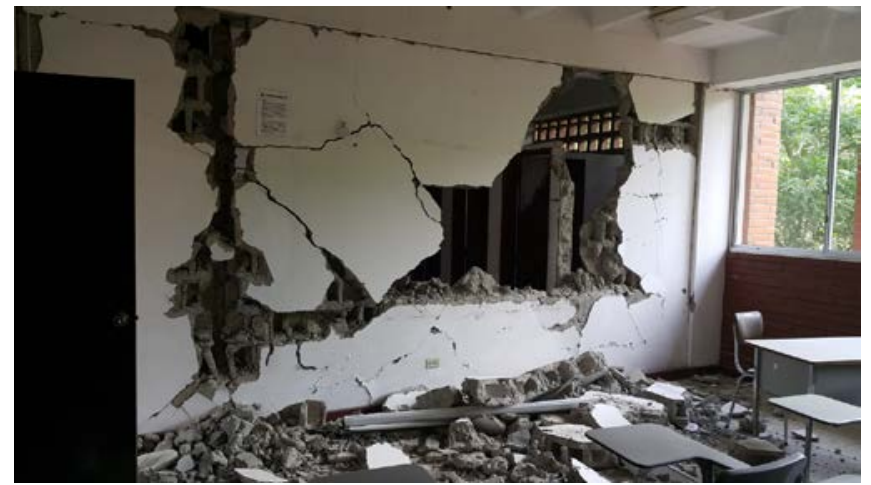

Fig. 1. Fallas en la mampostería por el sismo de 16 de abril de 2016

Su abundancia provocó diferentes estudios. Por ejemplo, en el trabajo de Jorge Silva [1] se analiza una muestra de 10 residencias de hormigón armado de 2 y 4 pisos con características tradicionales tales como losas alivianadas con vigas embebidas, columnas relativamente esbeltas y, sobre todo, con mampostería confinada hecha con bloques alivianados de baja calidad.

Los modelos analíticos mostraron que este tipo de estructuras no son capaces de soportar las cargas sísmicas requeridas por la norma ecuatoriana de la construcción actual, NEC-15 [2], si es que no se considera la contribución de la mampostería. Se concluye que el esqueleto de hormigón armado es excesivamente flexible y que colapsaría si no existiera el apoyo de la mampostería. Es decir, que ésta, por la forma de construir en el país, tiene un valor estructural.

Sin embargo, por su mala calidad, se daña mucho, tanto en extensión como en el grosor de fisuras y grietas. En particular, la deriva a partir de la cual ya se observa daño es de 0.005. Una alternativa para disminuir la cantidad de daño es rigidizar la estructura. Puesto que el $90 \%$ de las edificaciones en hormigón armado del país tienen esta tipología vulnerable, este reforzamiento es esencial. Pero, en primer lugar, ¿cómo determinar la facilidad o no de sufrir daño, y por tanto el tipo de reforzamiento necesario?

La extensión del daño esperado se puede estimar de manera aproximada por medio de relaciones tales como el alto de la estructura respecto a su periodo de vibración fundamental, $\mathrm{H}$ [m]/T[seg]. Estudios realizados en Chile [3] muestran que si ésta está entre 20 y 40, la edificación es flexible y por tanto se espera mucho daño. El parámetro clave en el cociente anterior es el periodo de vibración y por tanto debe estimarse de la forma más precisa posible. En efecto, ya se ha indicado, por ejemplo, que los modelos analíticos deben incluir la mampostería, lo que afecta en menos a estos períodos. Sin embargo, la rigidez de la 
mampostería es difícil de estimar, pues su comportamiento es no lineal y además está afectada por su forma de construcción.

Este artículo propone estimar la vulnerabilidad estructural a partir de la relación $\mathrm{H} / \mathrm{T}$, donde el periodo de vibración se obtiene experimentalmente. Aquí se describirá una metodología basada en los equipos y software disponibles en el país. Vale la pena indicar que el costo de los acelerómetros es un impedimento a la hora de difundir esta técnica de la manera más amplia posible. Por tanto, en este artículo también se propone el uso de acelerómetros basados en la tecnología Arduino, que reduce costos sustancialmente.

Las relaciones $\mathrm{H} / \mathrm{T}$ obtenidas para una muestra de residencias tipo se describen en la sección II. Luego, el fundamento teórico para la obtención de períodos de vibración a partir de registros acelerográficos se encuentra en la sección III. La metodología de medición de registros acelerográficos y su tratamiento se describen con un caso práctico en la Seccion IV. Los resultados se leen en la sección $\mathrm{V}$. El acelerómetro Arduino es descrito en la sección VI. Finalmente, conclusiones relevantes son presentadas en la sección VII.

\section{VALORES H/T EN RESIDENCIAS DEL ECUADOR}

La Tabla I muestra alturas y períodos de vibración de 10 residencias de 2 y 4 pisos de alto. Nótese que se muestran los períodos sin y con mampostería y los correspondientes valores, $\mathrm{H} / \mathrm{T}$ sin y $\mathrm{H} / \mathrm{Tcon}$.

Vale la pena indicar que el modelo analítico considera las vigas y columnas con una reducción en la rigidez de 50 y $20 \%$ respectivamente. Las vigas embebidas tienen dimensiones equivalentes, sumando las dimensiones del primer nervio adyacente de losas nervadas bidireccionales. Estas losas asumen comportamiento de membrana. Las estructuras se consideran empotradas a nivel de la calle. Para las mamposterías se ha considerado el modelo del puntal con las características descritas en FEMA 356 (2000) [5]. Los puntales se encuentran tanto en una dirección como en la opuesta en vanos con paneles completos de mampostería. Los valores de carga permanente, usados según NEC-15 para calcular la masa, incluyen el peso propio de la estructura, y cargas adicionales, por los acabados de construcción y mamposterías, de 300 kgf/m2.

TABLA I

VALORES H/T PARA UNA MUESTRA DE RESIDENCIAS EN ECUADOR.

\begin{tabular}{cccccc}
\hline \hline & H [m] & Tsin [seg] & H/Tsin & Tcon [seg] & H/Tcon \\
\hline 1. & 5.36 & 0.47 & 11 & 0.29 & 18 \\
2. & 5.28 & 0.548 & 10 & 0.433 & 12 \\
3. & 5.44 & 0.45 & 12 & 0.279 & 19 \\
4. & 6.23 & 0.439 & 14 & 0.196 & 32 \\
5. & 5.22 & 0.336 & 16 & 0.211 & 25 \\
6. & 7.94 & 0.647 & 12 & 0.449 & 17 \\
7. & 10.36 & 0.827 & 13 & 0.382 & 27 \\
$\mathbf{8 .}$ & 10.24 & 0.685 & 15 & 0.516 & 20 \\
9. & 10.57 & 0.567 & 19 & 0.371 & 28 \\
10. & 10.60 & 0.709 & 15 & 0.541 & 20 \\
\hline \hline
\end{tabular}

Se conoce, a partir de resultados analíticos, que las edificaciones de la muestra son muy vulnerables ante sismos y que, si bien en su gran mayoría no colapsan, sí sufren daños extensos en las mamposterías [1]. En la Tabla I se observa que el cociente H/Tsin que describe este tipo de comportamiento está entre 10 y 19. 10 ó menos indicará daño excesivo y hasta probable colapso, en tanto que 20 ó más indicará daño moderado o leve. El cociente H/Tcon tendrá el comportamiento indicado anteriormente si está entre 18 y 29. 17 o menos indicará probable colapso, y 30 ó más, significará daño moderado o leve. En todo caso, se observa que los periodos son mucho mayores que los usualmente esperados. Adicionalmente, nótese que en este artículo aún no se ha considerado el efecto negativo de posibles irregularidades en planta y en elevación, que al producir esfuerzos adicionales, acelerarían la falla estructural.

\section{ObTención de PeRiodos de Vibración A PARTiR DE REGISTROS ACELEROGRÁfICOS}

En la sección anterior se mostraron resultados analíticos de períodos de vibración que varían mucho de acuerdo a los elementos estructurales, en este caso mamposterías, que se consideran o no. Otra forma de calcularlos sería de forma experimental. De esta forma, también se validarían los modelos analíticos.

Obsérvese que los períodos de vibración en edificaciones dependen de su rigidez y masa. Mientras más masa, mayor periodo, y mientras más rigidez, menor periodo. Usualmente unos pocos modos de vibrar controlan el comportamiento estructural, siendo el más importante el de periodo más largo, denominado fundamental. En forma aproximada es igual a 0.11 veces el número de pisos. También es posible obtenerlo a partir del análisis de las aceleraciones ambientales de la edificación. Es necesario sin embargo, que la frecuencia de muestreo de las aceleraciones sea por lo menos dos veces la de la frecuencia fundamental de la edificación que se desea medir. Las frecuencias son simplemente el inverso de los períodos. El modo fundamental de vibración es usualmente lineal, aumentando con la altura. El mejor lugar para captarlo, puesto que las aceleraciones son mayores, es en la terraza de la edificación. Para obtener un resultado claro de la frecuencia fundamental, la señal acelerográfica se somete a un proceso de "limpieza" con filtros que eliminan frecuencias más altas o bajas que la deseada. El tratamiento matemático estándar para obtener la frecuencia fundamental de vibración a partir del registro acelerográfico es la transformada rápida de Fourier (FFT) [4]. Mientras más tiempo tenga el muestreo, más claramente se observarán las frecuencias propias de la estructura.

\section{MEDiCiÓN Y TRATAMiENTO DE LOS REgistros ACELEROGRÁficos (CASO PrÁCTICO)}

Se medirá el periodo de vibración fundamental del edificio de la Cámara de la Industria de la Construcción, CAMICON, ubicado en Quito, sector Iñaquito.

Se trata de un edificio de 8 pisos de alto más un subsuelo. Está constituido por pórticos con vigas descolgadas y losas alivianadas bidireccionales en hormigón armado. Es un edificio para oficinas, tiene acabados de costos medios y abundantes paredes de bloque, a excepción del ducto del ascensor donde son de ladrillo [5].

La medición se realiza con un acelerómetro tipo Libelium 
Plug and Sense. Se conecta directamente a un computador para obtener su máxima capacidad de registro que es de 50 $\mathrm{Hz}$. Se lo coloca en el centro de masas de la terraza y se lo opera por una hora. Un modelo analítico inicial de la edificación mostró un periodo fundamental de alrededor de 1 segundo, que se usa de referencia para escoger el número de registros y el tipo de filtros que se usarán en el tratamiento de la señal. Usando este dato, se trabaja con una frecuencia de muestreo de 10 puntos por segundo, ligeramente mayor que dos veces el número de puntos necesario para registrar completamente una señal con un periodo de 1 seg (4 puntos). Además se filtran los períodos menores a 0.3 y mayores a $5 \mathrm{~Hz}$ usando el método de Chebyshev, pues es el que da los resultados más claros. Los registros se manejan con el programa Seismosignal.

\section{ANÁlisis DE RESUltados}

La transformada rápida de Fourier tipo que se obtiene del proceso descrito anteriormente se tiene en la Figura 2.

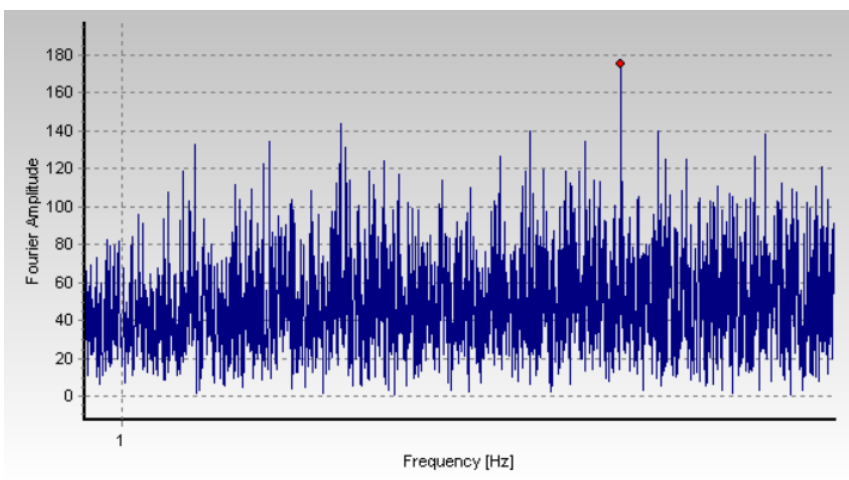

Fig. 2. FFT obtenida en el programa Seismosignal

Se observa una gran cantidad de picos, que representan ruido, pero también un valor máximo que se diferencia claramente y que corresponde a la frecuencia fundamental de vibración de la edificación.

Nótese que del registro inicial se extrajeron un total de 10 señales, 5 en sentido del largo en planta del edificio y 5 en sentido perpendicular. Cada señal tiene 4 "versiones", de acuerdo a la forma en que se tomen datos para obtener 10 puntos por segundo. Estas "versiones" tienen una extensión de 32768 puntos cada una y, como ya se indicado, una frecuencia de muestreo de 10 veces por segundo. La extensión de las "versiones" se debe a la capacidad del software Seismosignal [6].

Los resultados se observan en la Tabla II. En la Tabla II se observa que hay algunas "versiones" que se alejan de los valores que se obtienen normalmente. Se considera que esto se debe a la existencia de una componente de vibración por torsión en planta.

Sin embargo, al usar simplemente valores promedio, se tiene que el periodo de vibración en el sentido largo de la edificación, denominado sentido $\mathrm{X}$, es de 0.75 seg, $\mathrm{y}$ en sentido perpendicular, sentido $\mathrm{Y}$, es de $1.10 \mathrm{seg}$.

Utilizando este último valor, la relación H/T es 29 m/1.1 $\operatorname{seg}=26.4$.
TABLA II

FRECUENCIAS (1/T) Y PERÍODOS DE VIBRACIÓN (T) EN REGISTROS OBTENIDOS EN LA CAMICON (FUENTE: CEVALLOS Y BURBANO 2018)

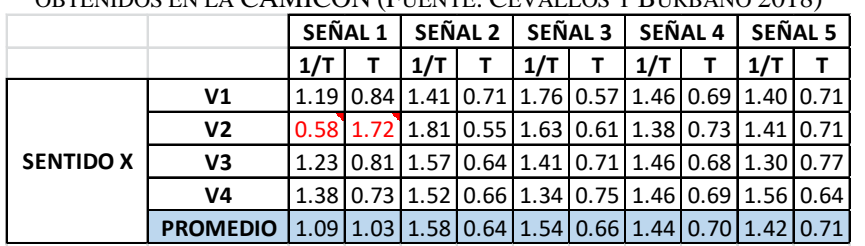

\begin{tabular}{|c|c|c|c|c|c|c|c|c|c|c|c|}
\hline & & \multicolumn{2}{|c|}{ SEÑAL 1} & \multicolumn{2}{|c|}{ SEÑAL 2} & \multicolumn{2}{|c|}{ SEÑAL 3} & \multicolumn{2}{|c|}{ SEÑAL 4} & \multicolumn{2}{|c|}{ SEÑAL 5} \\
\hline & & $1 / T$ & $T$ & $1 / \mathrm{T}$ & $\mathbf{T}$ & $1 / T$ & $\mathbf{T}$ & $1 / \mathrm{T}$ & $\mathbf{T}$ & $1 / T$ & $\mathbf{T}$ \\
\hline \multirow{5}{*}{ SENTIDO Y } & V1 & 1.41 & 0.71 & 1.62 & 0.62 & 1.54 & 0.65 & 0.35 & 2.83 & 1.51 & 0.66 \\
\hline & V2 & 1.63 & 0.62 & 1.33 & 0.75 & 1.47 & 0.68 & 1.59 & 0.63 & 1.49 & 0.67 \\
\hline & V3 & 0.36 & 2.75 & 1.45 & 0.69 & 1.56 & 0.64 & 0.51 & 1.96 & 0.55 & 1.82 \\
\hline & V4 & 1.75 & 0.57 & 1.45 & 0.69 & 1.61 & 0.62 & 1.47 & 0.68 & 0.37 & 2.72 \\
\hline & PROMEDIO & 1.29 & 1.16 & 1.46 & 0.69 & 1.55 & 0.65 & 0.98 & 1.53 & 0.98 & 1.47 \\
\hline
\end{tabular}

\section{El Dispositivo ARduino UnO}

En este artículo se ha mostrado la posibilidad de estimar la vulnerabilidad estructural a partir del cociente H/T. El parámetro decisivo en este cociente es $\mathrm{T}$, es decir el periodo de vibración de la estructura, que aquí se ha determinado de forma experimental. Sin embargo, el costo de los acelerómetros que se usan en este procedimiento, puede ser un impedimento a la hora de generalizar su aplicación.

Afortunadamente, existe la tecnología Arduino que reduce sustancialmente estos costos [7]. El dispositivo consiste de tres partes, como se observa en la Figura 3: el acelerómetro (a la izquierda), un tablero de conexión y el sistema que maneja la señal del acelerómetro para enviarla a un computador (a la derecha).

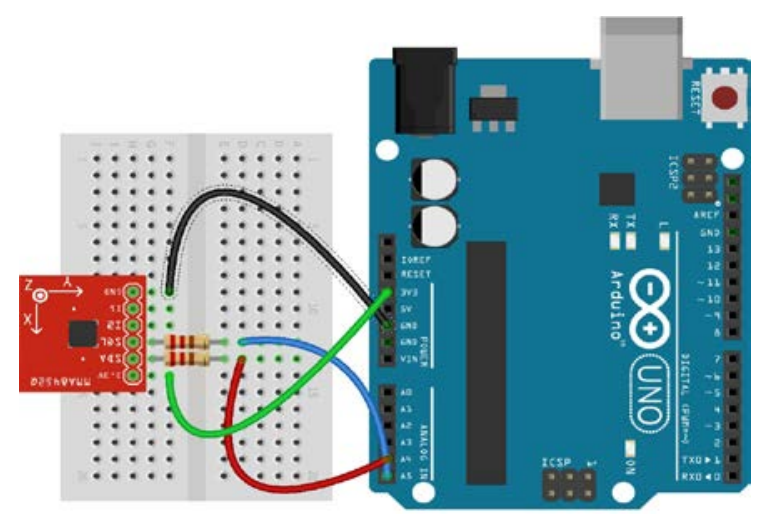

Fig. 3. Partes del dispositivo Arduino

El acelerómetro es capaz de captar señales tanto para dos ejes horizontales como para un eje vertical. Puede cambiarse de acuerdo a las necesidades de mayor o menor sensibilidad.

Este dispositivo se conecta a una computadora que lee las señales mediante el programa Arduino IDE. Este puede descargarse de https:/www.arduino.cc/en/Main/Software. Además, es necesario instalar el archivo de librerías del acelerómetro, y seleccionar la opción de graficación "Serial Plotter”, “115200 baud” [8].

En Ecuador, este dispositivo puede conseguirse a un precio de alrededor de 40 usd [7].

\section{CONCLUSIONES}

El cociente, altura de la edificación sobre periodo de vibración, $\mathrm{H} / \mathrm{T}$, obtenido en una muestra de 10 residencias de entre 2 y 4 pisos, muestra que un comportamiento con daño muy extenso en mamposterías, estaría definido por 
valores entre 18 y 29. Este resultado es semejante al sugerido por estudios similares en Chile [3]. Sin embargo, la modelación analítica de la mampostería es relativamente complicada. Una forma alternativa de calcular el periodo de vibración fundamental de una edificación es por medio de acelerómetros. Basta con una señal obtenida en la terraza de la edificación, por ser el sitio con aceleraciones más altas. La claridad de la señal puede mejorar con un mayor tiempo de captación y mediante el uso de diferentes filtros analíticos. La transformada rápida de Fourier, muestra, a pesar de todo, ruido, pero también claramente la frecuencia de vibración fundamental de la edificación. El procedimiento que se ha descrito en este artículo usa equipo y software ya disponibles en el Ecuador. Se observó, además, que este procedimiento permite determinar de manera rápida la vulnerabilidad de una edificación. Los resultados obtenidos en este artículo, muestran una dispersión relativamente pequeña, lo que indica su confiabilidad. Un cociente tal como $\mathrm{H} / \mathrm{T}$, obtenido de forma experimental, podría incluirse de forma obligatoria en el análisis estructural de edificaciones, como un parámetro para determinar la suficiencia o no de un diseño sismorresistente. Finalmente, para facilitar la generalización de esta metodología, se podrían usar acelerómetros tipo Arduino, por su bajo costo.

\section{REFERENCIAS}

[1] J. Silva, "Guía de reforzamiento para estructuras informales aporticadas según estudio de vulnerabilidad en el Distrito Metropolitano de Quito”, trabajo de titulación previo a la obtención del título de ingeniero civil, Universidad de las Fuerzas Armadas ESPE, 2017.

[2] NEC-SE-DS, "Norma Ecuatoriana de la Construcción - Seguridad Estructural - Diseño Sismo Resistente”, Quito: Ministerio de Desarrollo Urbano y Vivienda, 2015.

[3] T. Guendelman, J. Lindenberg, M. Guendelman, "Perfil Bío-Sísmico de edificios”, Proc. Séptimas jornadas chilenas de sismología e ingeniería antisísmica, 1997.

[4] FFT, Mathworld, ttp://mathworld.wolfram.com/FastFourierTransform.html, acceso 29 de abril de 2018.

[5] A. Cevallos, A. Burbano, trabajo de titulación previo a la obtención del título de ingeniero civil, Universidad de las Fuerzas Armadas ESPE, en preparación, 2018.

[6] Seismosignal, software estructural, www.seismosoft.com/seismosignal, acceso 29 de abril de 2018.

[7] R. Moreano, trabajo de titulación previo a la obtención del título de ingeniero civil, Universidad de las Fuerzas Armadas ESPE, en preparación, 2018.

[8] Arduino IDE, https://www.arduino.cc/en/Main/Software, acceso 29 de abril de 2018. 